\title{
The aggregation kinetics and filament structure of a tau fragment are influenced by the sulfation pattern of the cofactor heparin
}

David Townsenda ${ }^{a}$, Nigel J. Fullwood ${ }^{b}$, Edwin A. Yates ${ }^{c}$, David A. Middleton ${ }^{a *}$

aDepartment of Chemistry, University of Lancaster, Lancaster LA1 4YB, U.K.

bDivision of Biomedical and Life Sciences, University of Lancaster, Lancaster LA1 4YG, U.K.

'Department of Biochemistry and Systems Biology, Institute of Systems, Molecular and Integrative Biology, University of Liverpool, L69 7ZB, U.K.

*Address correspondence to:

David A. Middleton

Department of Chemistry, University of Lancaster, Lancaster LA1 4YB, U.K.

E-mail: d.middleton@lancaster.ac.uk

Tel: +44 1524594328 


\section{Abstract}

A pathological signature of Alzheimer's disease (AD) is the formation of neurofibrillary tangles comprising filamentous aggregates of the microtubule associated protein tau. Tau self-assembly is accelerated by polyanions including heparin, an analog of heparan sulfate. Tau filaments co-localise with heparan sulfate proteoglycans (HSPGs) in vivo and HSPGs may also assist the transcellular propagation of tau aggregates. Here, we investigate the role of the sulfate moieties of heparin in the aggregation of a recombinant tau fragment $\Delta$ tau187, comprising residues $255-441$ of the C-terminal microtubule-binding domain. The effects that the selective removal of the $\mathrm{N}$-, 2-O- and 6-O-sulfate groups from heparin have on the kinetics of tau aggregation, aggregate morphology and protein structure and dynamics were examined. Aggregation kinetics monitored by thioflavin T (ThT) fluorescence revealed that aggregation is considerably slower in the presence of 2O-desulfated heparin than with $\mathrm{N}$ - or 6-O-desulfated heparin. Transmission electron microscopy revealed that tau filaments induced by 2-O-desulfated heparin were more slender than filaments formed in the presence of intact heparin or 6-Odesulfated heparin. The 2-O-desulfated heparin-induced filaments had more extensive regions of flexibility than the other filaments, according to circular dichroism and solid-state NMR spectroscopy. These results indicate that the sulfation pattern of heparin regulates tau aggregation, not purely though electrostatic forces but also through conformational perturbations of heparin when the 2-O-sulfate is removed. These findings may have implications for the progression of $A D$, as the sulfation pattern of GAGs is known to change during the ageing process, the main risk factor for the disease. 


\section{Introduction}

The assembly of microtubule-associated protein tau (MAPT, or tau; UniProtKB P10636) into filamentous aggregates is a pathological hallmark of neurogenerative diseases including Alzheimer's disease (AD) and Pick's disease, collectively known as tauopathies.1, 2 Tau is a water-soluble, intrinsically disordered protein under normal physiological conditions and its function is to assist the assembly and stabilisation of microtubules and other neuronal cytoskeletal elements. Six isoforms of tau occur in the adult human brain; ${ }^{3}$ the largest (441-aa) isoform consists of two $\mathrm{N}$-terminal inserts (N1, $\mathrm{N} 2)$ and four repeat units (R1-R4) in the microtubule-binding C-terminal region. The shorter isoforms lack one or both of the $\mathrm{N} 1$ and $\mathrm{N} 2$ units and/or the R2 unit. Neurofibrillary tangles of abnormally phosphorylated, aggregation-prone tau occur in the brains of patients affected by $A D$, and these consist predominantly of insoluble paired helical filaments (PHF) and straight filaments (SF).4, 5 The filaments consist of an ordered $\beta$-sheet amyloid core flanked by a fuzzy, unstructured coat of around 200 residues that is invisible to transmission electron microscopy (TEM). ${ }^{6}$ Recent visualisation by electron cryo-microscopy, supported by earlier solid-state NMR studies, ${ }^{7}$, 8 have revealed that the nanoscale polymorphism of tau PHF and SF originates from disease-specific molecular conformations of tau within the fibres, ${ }^{5,9}$ raising the possibility that neuropathological strains may propagate in a prion-like mechanism. The molecular processes that lead to the formation and propagation of these strains in vivo are far from clear and our understanding has until recently depended upon mechanistic studies in vitro.

Studies of the aggregation mechanisms of intact or truncated tau proteins in vitro require the addition of arachidonic acid or polyanionic cofactors such as RNA and, most commonly, heparin to initiate aggregation. ${ }^{10,11}$ Recent results from tissuederived filaments pose the question of whether the heparin-induced tau aggregation is pathologically relevant. ${ }^{12}, 13$ Heparin-induced filaments are heterogeneous, ${ }^{13}$ possibly reflecting the heterogeneity and polydispersity of the heparin cofactor, and the protein molecular conformation differs somewhat from the structures associated with $A D$ or Pick's. It is worth noting, however, that there is insufficient information about the structural variability of filaments obtained from different $A D$ or Pick's brains, given the limited current data and challenges involved. Further, heparin is closely related to heparan sulfate, which was found early on to accumulate with 
neurofibrillary tangles in the $A D$ brain ${ }^{14}$ and, when conjugated to proteoglycans, assist the cellular uptake and possible propagation of tau via a prion-like seeding mechanism ${ }^{15}$.

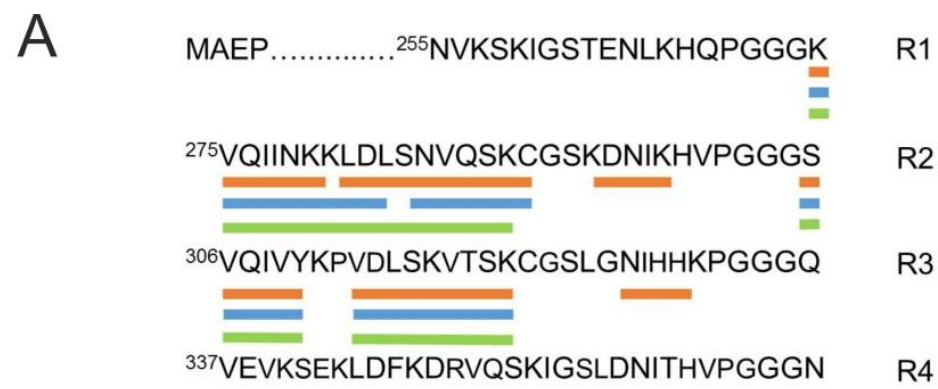

KQGL $^{441}$

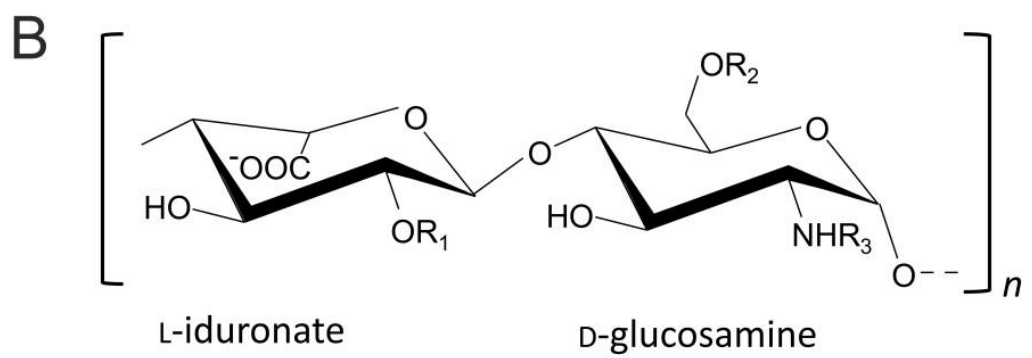

\begin{tabular}{l|clll} 
& $n$ & $\mathrm{R}_{1}$ & $\mathrm{R}_{2}$ & \multicolumn{1}{c}{$\mathrm{R}_{3}$} \\
\hline LMWH & 9 & $\mathrm{SO}_{3}^{-}$ & $\mathrm{SO}_{3}^{-}$ & $\mathrm{SO}_{3}^{-}$ \\
LMW-6OH & 9 & $\mathrm{SO}_{3}^{-}$ & $\mathrm{H}$ & $\mathrm{SO}_{3}^{-}$ \\
LMW-2OH & 9 & $\mathrm{H}$ & $\mathrm{SO}_{3}^{-}$ & $\mathrm{SO}_{3}^{-}$ \\
LMW-NAc & 9 & $\mathrm{SO}_{3}^{-}$ & $\mathrm{SO}_{3}^{-}$ & $\mathrm{COCH}_{3}$ \\
LMW-6OH-NAc & 9 & $\mathrm{SO}_{3}^{-}$ & $\mathrm{H}$ & $\mathrm{COCH}_{3}$ \\
LMW-2OH-NAc & 9 & $\mathrm{H}$ & $\mathrm{SO}_{3}^{-}$ & $\mathrm{COCH}_{3}$ \\
LMW-2OH-6OH & 9 & $\mathrm{H}$ & $\mathrm{H}$ & $\mathrm{SO}_{3}^{-}$ \\
LMW-2OH-6OH-NAc & 9 & $\mathrm{H}$ & $\mathrm{H}$ & $\mathrm{COCH}_{3}$ \\
HMWH & $12-16$ & $\mathrm{SO}_{3}^{-}$ & $\mathrm{SO}_{3}^{-}$ & $\mathrm{SO}_{3}^{-}$ \\
HMW-6OH & $12-16$ & $\mathrm{SO}_{3}^{-}$ & $\mathrm{H}$ & $\mathrm{SO}_{3}^{-}$ \\
HMW-I2OH & $12-16$ & $\mathrm{H}$ & $\mathrm{SO}_{3}^{-}$ & $\mathrm{SO}_{3}^{-}$ \\
HMW-NAc & $12-16$ & $\mathrm{SO}_{3}^{-}$ & $\mathrm{SO}_{3}^{-}$ & $\mathrm{COCH}_{3}$
\end{tabular}

Scheme 1. (A) Sequence of the four microtubule binding repeats (R1-R4) and $\beta$ strand regions of the heparin-induced 2N4R tau polymorphs (orange, snake; blue, twisted; green, jagged) identified by cryo-EM. (B) Generic structure of the heparin disaccharide unit and list of the desulfated heparin derivatives prepared.

It has often been assumed that heparin-tau interactions are driven largely by non-specific ionic interactions between the protein and the sulfate and carboxylate groups of heparin, ${ }^{16}$ but this notion has been challenged by recent results showing that the sulfation pattern of HS influences the cellular internalisation of tau, with the 6-O-sulfate being critical for this process and for tau binding. ${ }^{17-19}$ Here, using 
selectively desulfated heparin molecules, we show that the sulfation pattern of heparin also influences the rate of aggregation of a truncated tau construct and modulates the mass, nanoscale morphology and atomic structure of the filaments formed. All experiments were performed with the $\Delta$ tau187 construct $^{20}$ comprising residues 255-441 of the tau N2R4 isoform, spanning repeat units R1-R4 and encompassing the amyloid core region from N256 to F378 (Scheme 1A). Porcine mucosal low-molecular weight heparin (LMWH; approximately $6 \mathrm{kDa}, \mathrm{dp} 18)$ and high molecular weight heparin (HMWH; approximately $16 \mathrm{kDa}$, dp24-32) were chemically modified to remove the 2-O-sulfate groups and/or the 6-O-sulfate groups and/or replace the $\mathrm{N}$-sulfates with acetyl groups, yielding 10 derivatives (Scheme 1B). The residual sulfate levels of the 6-O- and 2-O- desulfated LMW heparin derivatives are $61 \%$ and $70 \%$, and, of the HMW heparin derivatives are $50 \%$ and $42 \%$, of the sulfate levels of the respective unmodified heparin, as measured by enzymatic digestion followed by high-performance anion exchange chromatography (HPAEC).

\section{Experimental methods}

\section{Tau expression}

The tau construct comprising residues 255-441 of human 4R tau (cDNA clone htau46) with the aggregation impeding $N$ terminus removed, leaving the $2^{\text {nd }}$ and $3^{\text {rd }}$ repeat microtubule binding (MTB) units, including the highly amyloidogenic sequences VQIINK and VQIVYK, respectively. The protein was expressed and purified as previously described. ${ }^{20}$

\section{Preparation of desulfated heparin}

Unmodified low molecular weight heparin (LMWH) (approx. dp 18) and high molecular weight heparin (approx. $36 \mathrm{dp}$ ) were purchased from Iduron (Manchester, UK). Modified heparin derivatives were prepared through appropriate combinations of solvolytic de-sulfation ${ }^{21,22}$, involving the removal of iduronate 2-O-sulfation via 2,3epoxide formation and subsequent nucleophilic ring opening ${ }^{23}$ and $\mathrm{N}$-acetylation, followed by purification. Purification involved, for the 6 -de-O-sulfated derivatives, a precipitation step in ethanol, followed by dialysis (3.5 kDa cut-off, $2 \mathrm{~L} \times 3$ ), gel permeation chromatography (Sephadex G-25), cation exchange (Dowex-50 Na+ 
form) and then freeze-drying. For the 2-O-de-sulfated derivatives, purification involved a dialysis step (3.5 kDa cut-off, $2 \mathrm{~L} \times 3$ ) followed by GPC (Sephadex G-25) and cation exchange (Dowex-50 Na+ form), prior to freeze-drying. The compounds were characterised by ${ }^{1} \mathrm{H}$ and ${ }^{13} \mathrm{C}$ NMR spectroscopy in $\mathrm{D}_{2} \mathrm{O}$, essentially as described, ${ }^{24}$ to establish the purity and the extent of modification by reference to established chemical shift positions. The extent of modification was measured by analysis of the HPAEC (Propac PA-1 column $2 \times 250 \mathrm{~mm}$ ) profiles (monitoring the double bond formed by lyase treatment at $232 \mathrm{~nm}$ ) of complete digestion products following bacterial (Flavobacterium heparinium) heparinase digestion (hl, hll and hlll) in lyase buffer ( $50 \mathrm{mM}$ sodium acetate, $2.5 \mathrm{mM}$ calcium acetate) and reference to standard disaccharides (Dextra Labs, UK) to provide the proportions of each constituent disaccharide to be established and the extent of particular substitutions to be calculated with reference to the values obtained for the relevant (LMW or HMW) heparin starting material.

\section{Aggregation kinetics of tau}

The formation of amyloid was measured with the amyloid specific dye Thioflavin $T$ (ThT). Tau with heparin (20 and $5 \mu \mathrm{M}$ respectively), were incubated with Tris (30 $\mathrm{mM})$, DTT $(1 \mathrm{mM})$ at $\mathrm{pH} 7.5$ in the presence of $20 \mu \mathrm{M}$ Thioflavin at $37{ }^{\circ} \mathrm{C}$. Fluorescence measurements, with excitation at $450 \mathrm{~nm}$ and emission at $482 \mathrm{~nm}$, were taken from triplicate samples on a Molecular Devices Flexstation 3 Microplate Reader (Molecular Devices), every 2 minutes for up to $24 \mathrm{~h}$ with shaking for 10 seconds prior to each read.

\section{Circular dichroism}

Tau $(20 \mu \mathrm{M})$ was incubated with Tris $(30 \mathrm{mM})$, DTT $(1 \mathrm{mM})$ at $\mathrm{pH} 7.5$ with heparin derivates $(5 \mu \mathrm{M})$ at $37^{\circ} \mathrm{C}$ with agitation. Spectra were acquired hourly during the first 8 hours, followed by acquisition of a final spectrum after 24 hours. Spectra were acquired on a Chirascan Plus CD spectrometer between 180 and $260 \mathrm{~nm}$ with a band width of $1 \mathrm{~nm}$, using a path-length of $0.1 \mathrm{~mm}$. Background signals of buffer, and the relevant heparin derivative were removed from the spectra. Measurements were collected from triplicate samples, and the averaged spectra were fitted with the 
BestSel algorithm between 190-250 nm, and percentage of helical, $\beta$-sheet, turn and unordered components were calculated.. 25

\section{Transmission Electron Microscopy}

Tau and heparin (20 and $5 \mu \mathrm{M}$, respectively) were incubated with Tris (30 mM), DTT $(1 \mathrm{mM})$ at $\mathrm{pH} 7.5$ at $37^{\circ} \mathrm{C}$ for 24 hours. A $10 \mu \mathrm{L}$ suspension was spotted onto carbon coated formar grids. After 5 minutes the excess liquid was removed via blotting. For negative staining, $10 \mu \mathrm{L}$ of $2 \%$ phosphotungstic acid was spotted onto the loaded grids, and left for 3 minutes before blotting the excess. Grids were viewed on a Jeol JEM-1010 electron microscope and images captured that were representative of the entire grid. Fibril widths were measured using ImageJ software and the averages from 50 measurements was calculated.

\section{Solid-state NMR experiments}

Following 24 hours of agitated growth of tau $(20 \mu \mathrm{M})$ at $37^{\circ} \mathrm{C}$ in the presence of the heparin derivatives $(5 \mathrm{mM})$, tau aggregates were pelleted by centrifugation at 50000 $g$ for $1 \mathrm{~h}$ in an ultracentrifuge (Beckman Coulter) and the supernatant was removed. The fibrils were sedimented by a second centrifugation step. The pellet was separated from the supernatant and packed in its hydrated state by ultracentrifugation into a $3.2 \mathrm{~mm}$ zirconia MAS rotor without further treatment for analysis by SSNMR.

Two-dimensional ${ }^{13} \mathrm{C}-{ }^{13} \mathrm{C}$ SSNMR spectra were recorded at a magnetic field of 16.3 Tesla on a Bruker Avance III 700 spectrometer, with a $3.2 \mathrm{~mm} \mathrm{HXY} \mathrm{probe}$ operating in double resonance mode. The magic-angle frequency was $13 \mathrm{kHz}$. The operating temperature was $4{ }^{\circ} \mathrm{C}$. Hartmann-Hahn cross-polarization was achieved with a 2 ms contact time and $100 \mathrm{kHz}$ proton decoupling with SPINAL-64 was applied during signal acquisition. Spectra were recorded with either $10 \mathrm{~ms}, 50 \mathrm{~ms}$ or 200 ms mixing times during which the proton nutation frequency was adjusted to the MAS frequency of $14 \mathrm{kHz}$ to meet the dipolar-assisted rotational resonance (DARR) condition ${ }^{26}$. Typically, 480 increments were acquired in the indirect $\left(\mathrm{t}_{1}\right)$ dimension with 400-600 transients per increment, and the total measurement time varied from 2-7 days depending on the efficiency of rotor packing. Phase-sensitive detection in the indirect dimensions was achieved using the States-TPPI method. Chemical shifts are expressed relative to tetramethylsilane. 
The simulated ${ }^{13} \mathrm{C}-{ }^{13} \mathrm{C}$ spectrum (Supporting Information, Figure $\mathrm{S} 1$ ) was calculated from chemical shifts predicted from the cryo-EM structure of heparin induced 2 N4R tau snake filaments, ${ }^{12}$ using a $C$ program written specifically for this purpose.

\section{Results}

\section{Desulfation of heparin influences $\Delta$ tau187 aggregation kinetics}

Incubation of $\Delta \operatorname{tau} 187(20 \mu \mathrm{M})$ with unmodified LMWH in the presence of the amyloid-sensitive dye thioflavin $\mathrm{T}$ (ThT) indicates that tau aggregation reaches completion within $5 \mathrm{~h}$ and the aggregation kinetics follow a sigmoidal trajectory consistent with a lag phase, filament growth and maturation, with a half-time t0.5 of $0.5 \mathrm{~h}$ (Figure $1 \mathrm{~A}$ ). Removal of only the 6-O-sulfates of $\mathrm{LMWH}$, or selective replacement of the $\mathrm{N}$-sulfates with $\mathrm{COCH}_{3}$, (Figure 1B) has a measureable effect on the aggregation kinetics, increasing to.5 to $1.4 \mathrm{~h}$ in both cases. A considerably slower rate of aggregation is seen in the presence of selectively 2-O-desulfated heparin (to.5 $>6 \mathrm{~h}$ ). Removal of the 6-Osulfate groups combined with $\mathrm{N}$-acetylation increases to.5 to $2.4 \mathrm{~h}$, but aggregation is much slower when 2-O-desulfation is combined with either 6-O-desulfation or $\mathrm{N}$ acetylation (to.5 $=4.5 \mathrm{~h}$ ) (Figure $1 \mathrm{C})$. Aggregation kinetics in the presence of $\mathrm{HMWH}$ are also dependent on the sites of heparin desulfation, with $\Delta$ tau187 aggregation again being considerably slower in the presence of 2-O-desulfated heparin than with the other derivatives (Figure 1D). In summary, the overall rate of heparin-induced $\Delta$ tau187 aggregation to completion is impeded to a greater extent by removing the 2-O-sulfates of heparin than it is by removing the $6-\mathrm{O}$ - or $\mathrm{N}$-sulfates. Although the data for $\Delta$ tau187 is normalized to the final fluorescence emission that was measured, the curve is clearly continuing in an upward trajectory, indicating that aggregation had not reached its conclusion even after $12 \mathrm{~h}$, and to. 5 is likely to be considerably longer than $6 \mathrm{~h}$. The different effect of the selectively desulfated heparin derivatives argues against the tauheparin interaction being mediated only by non-specific charge interactions. Other chemical and/or conformational properties must be considered. 
A

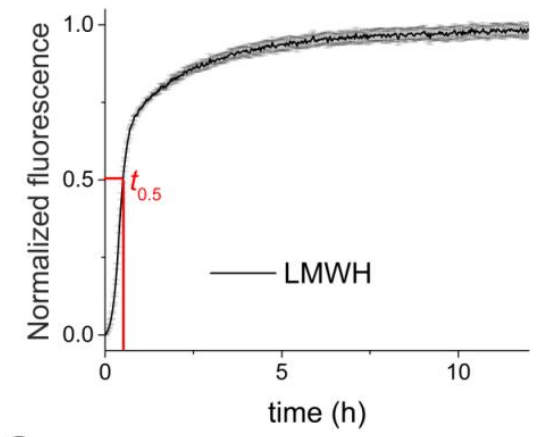

C

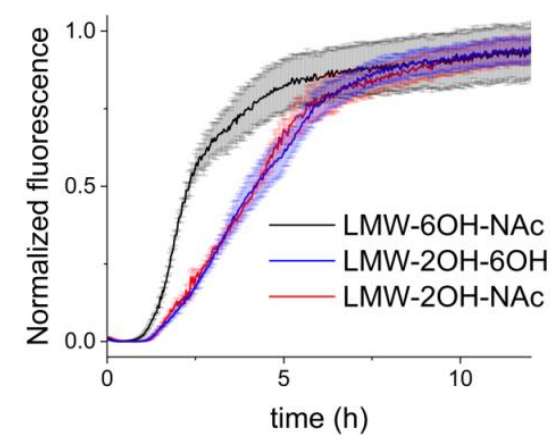

B

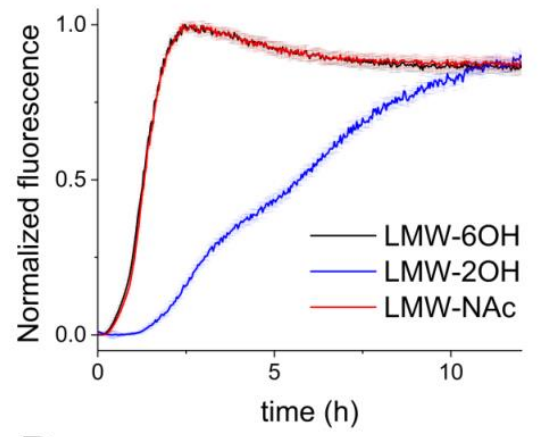

D

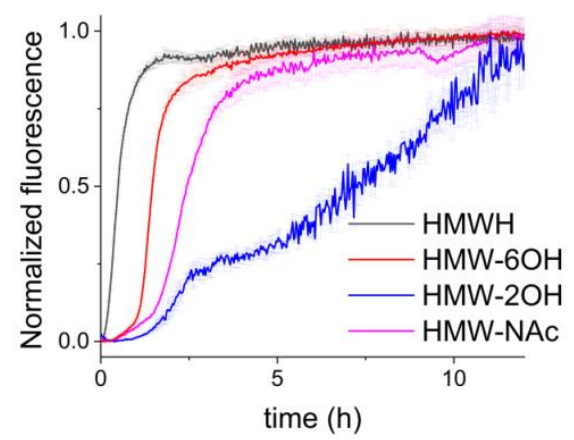

Figure 1. Aggregation kinetics of $\Delta \operatorname{tau} 187(20 \mu \mathrm{M})$ in the presence of unmodified heparin $(5 \mu \mathrm{M})$ monitored by ThT fluorescence. (A) Unmodified and fully desulfated LMWH. (B) LMWH desulfated at a single site per disaccharide unit. (C) LMWH desulfated at two sites per disaccharide unit. (D) Unmodified HMWH and HMWH desulfated at one site per disaccharide. Means and error bars are shown for triplicate measurements.

\section{Concentration dependence of desulfated heparin-induced $\Delta$ tau187 aggregation}

To further investigate the effect of the sulfate groups of heparin on $\Delta \operatorname{tau} 187$ aggregation, the kinetics were followed (by ThT) at different concentrations of LMWH or the 6-O, $\mathrm{N}$-acetylated and 2-O-desulfated derivatives (Figure 2). The time to completion of aggregation (quantified by to.5) is sensitive to heparin concentration, as is the ThT fluorescence at the end point of aggregation, suggesting that the mass of filaments is heparin concentration dependent. Curves calculated for the apparent rate constants for primary nucleation $\left(k_{n}\right)$, elongation $\left(k_{+}\right)$and secondary nucleation $\left(k_{2}\right),{ }^{27,28}$ agree well with the experimental data (Figure 2 and Table 1). The aggregation rate in the presence of unmodified LMWH remains constant up to $10 \mu \mathrm{M}$ heparin (to.5 $0.5 \mathrm{~h}$ ), with the final fibril mass scaling approximately with heparin concentration. The end-point ThT fluorescence also increases with heparin concentration up to $10 \mu \mathrm{M}$. This trend has been attributed to an increase in the fibrillar mass with increasing heparin concentration, which gives rise to 
enhanced ThT fluorescence. ${ }^{29}$ Although it is known that many other environmental factors or conformational differences can influence ThT fluorescence, it is likely that here the ThT curves do indeed reflect the fibril mass, as the heparin concentration (which is not ThT reactive) is the only controlled variable. At an equimolar $(20 \mu \mathrm{M}) \mathrm{LMWH}$ and $\Delta$ tau187 concentration, the aggregation rate decreases markedly $(t .5=1.7 \mathrm{~h}$ ) and no further increase in ThT fluorescence (or end-point fibril mass) is observed (Figure 2, top left). The reduced rate can be attributed to a reduction in both the apparent $k_{n}$ and $k_{+}$ values (Table 1). The retarding effect of heparin at higher concentrations is known and has been attributed to a screening effect of high heparin concentrations because of increased ionic strength. ${ }^{30}$ It has also been proposed that high heparin concentrations promote the formation of dead-end, off-pathway tau-heparin complexes. ${ }^{31}$ A further explanation, which will be explored in the next section, is that higher heparin concentrations increase the number of 1:1 tau:heparin complexes that are below the critical mass necessary to nucleate filament growth.

LMWH

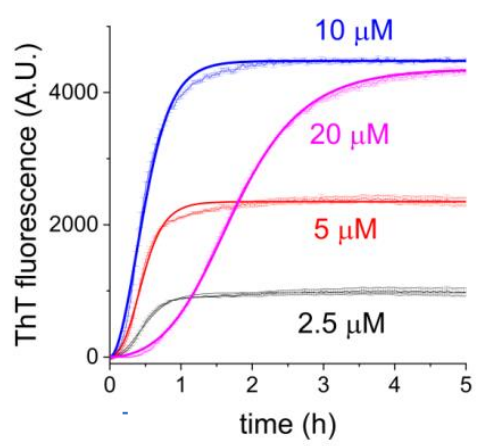

LMW-NAc

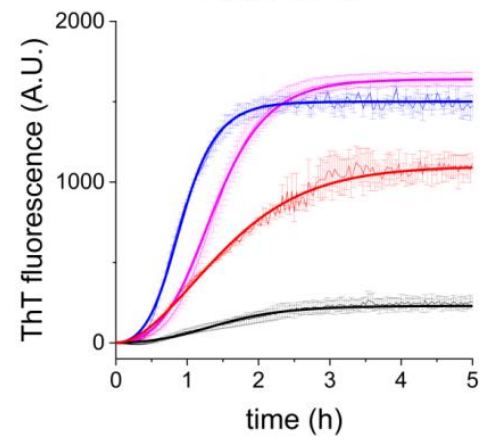

LMW-6OH

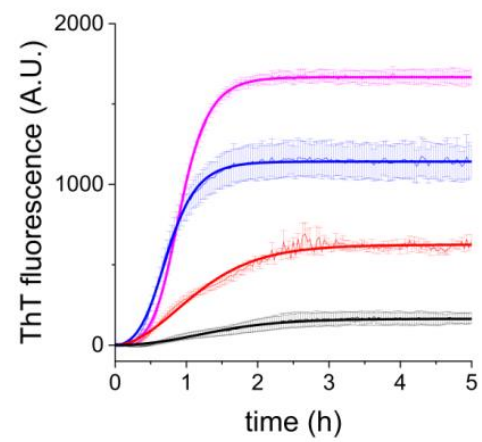

LMW-2OH

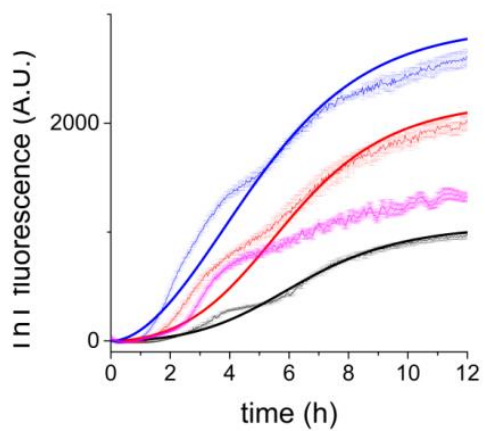

Figure 2. Aggregation kinetics of $\Delta \operatorname{tau} 187(20 \mu \mathrm{M})$ in the presence of LMWH or derivatives (desulfated at one site per disaccharide) at four concentrations, measured by thioflavin $T$ fluorescence. Means and standard errors are shown from triplicate measurements. Bold lines are the best fitting curves corresponding to the rate constants given in Table 1. Means and error bars are shown for triplicate measurements. 
When $\Delta$ tau187 is incubated with 6-O-desulfated or $\mathrm{N}$-acetylated LMWH, a 2-3-fold overall decrease in the rate of aggregation is seen relative to unmodified LMWH, with $t_{0.5}$ being 0.9 - $1.7 \mathrm{~h}$ (Figure 2, top right and bottom left; Table 1). Aggregation again follows a sigmoidal profile and the reduced rate appears to originate chiefly from a reduction in $k_{n}$ and $k_{+}$. Fluorescence at the conclusion of aggregation has a non-linear relationship with heparin concentrations up to $20 \mu \mathrm{M}$ heparin. In the presence of 2-O-desulfated LMWH, aggregation is substantially slower at all heparin concentrations, with $t_{0.5}$ in excess of $4 \mathrm{~h}$ and the growth curves are consistent with an apparent reduction of $k_{n}, k_{+}$and $k_{2}$ (Figure 2 , bottom right; Table 1) compared to the rate constant values in the presence of the other cofactors. Interestingly, the final aggregate mass decreases by $50 \%$ on increasing the 2-O-desulfated LMWH concentration from $10 \mu \mathrm{M}$ to $20 \mu \mathrm{M}$.

\section{Heparin 6-O-desulfation, but not 2-0-desulfation, reduces the affinity for $\Delta$ tau187}

The total residual sulfation levels of the 6-O- and 2-O-desulfated LMWH molecules are very similar (as are 6-O- and 2-O-desulfated $\mathrm{HMWH}$ ) and so the large differences in the behaviour of these desulfated heparins cannot be attributed entirely to their reduced charge density. Indeed, the ThT data reveal a complex relationship between the sulfation patterns and concentration of heparin, $\Delta$ tau187 aggregation rates and filament mass that cannot be explained fully by the apparent rate constants in Table 1. These rate constants describe a cofactor-independent aggregation mechanism represented by the equations, ${ }^{27,28}$

$\frac{d P(t)}{d t}=k_{2} m(t)^{n_{2}} M(t)+k_{n} m(t)^{n_{c}}$

and

$\frac{d M(t)}{d t}=2 k_{+} m(t) P(t)$

Here, $M(t)$ and $P(t)$ are the filament mass and number, respectively, at time $t$ and $\mathrm{m}(\mathrm{t})$ is the monomer concentration. The term $k_{n} m(t)^{n_{c}}$ represents the formation of primary nuclei from monomers $(m)$ with reaction order $n_{\mathrm{c}}$ (typically assigned a value of 2). The term $k_{2} m(t)^{n_{2}} M(t)$ describes secondary nucleation in a reaction of order $n_{2}$ (typically given a value of 2 or 3 ) with respect to the mass of monomer. The term 
Table 1. Summary of the apparent rate constants for tau aggregation in the presence of unmodified heparin and desulfated derivatives, obtained from the lines of best fit to the data in Figure 2. The calculations were also based on fixed values for the fibril dissociation rate constant $\left(k_{m}=1.0 \mathrm{M}^{-1} \mathrm{~s}^{-1}\right)$ and the reaction orders of primary nucleation $\left(n_{\mathrm{c}}=2\right)$ and secondary nucleation $\left(n_{2}=2\right) .{ }^{27,}{ }^{28}$ The reaction orders do not necessarily correspond to the size of the nuclei, but heparin has been shown to interact with two tau molecules forming a dimer that nucleates fibril growth. ${ }^{31}$ Dashes indicate where constants could not be calculated because of poor fits to the experimental data. Errors in to.5 are given in parentheses.

\begin{tabular}{|c|c|c|c|c|c|}
\hline Cofactor & $\begin{array}{l}\text { Conc. } \\
/ \mu \mathrm{M}\end{array}$ & $\begin{array}{c}k_{\mathrm{n}} \\
/ 10^{-6} \mathrm{M}^{-1} \mathrm{~s}^{-1}\end{array}$ & $\begin{array}{c}k_{+} \\
/ 10^{6} \mathrm{M}^{-1} \mathrm{~s}^{-1}\end{array}$ & $\begin{array}{c}k 2 \\
/ M^{-2} s^{-1}\end{array}$ & $\begin{array}{l}t 0.5 \\
/ \mathrm{h}\end{array}$ \\
\hline \multirow[t]{4}{*}{ LMWH } & 2.5 & 32 & 17 & 10 & $0.51(0.10)$ \\
\hline & 5.0 & 35 & 19 & 10 & $0.46(0.10)$ \\
\hline & 10.0 & 33 & 18 & 70 & $0.45(0.10)$ \\
\hline & 20.0 & 6 & 4 & 1 & $1.70(0.13)$ \\
\hline \multirow[t]{4}{*}{ LMW-6OH } & 2.5 & 4 & 7 & 13 & $1.47(0.24)$ \\
\hline & 5.0 & 6 & 9 & 12 & $1.12(0.14)$ \\
\hline & 10.0 & 9 & 15 & 16 & $0.72(0.12)$ \\
\hline & 20.0 & 6 & 11 & 16 & $0.95(0.06)$ \\
\hline \multirow[t]{4}{*}{ LMW-NAC } & 2.5 & 6 & 4 & 10 & $1.4(0.13)$ \\
\hline & 5.0 & 5 & 6 & 10 & $1.4(0.15)$ \\
\hline & 10.0 & 5 & 8 & 11 & $0.9(0.10)$ \\
\hline & 20.0 & 3 & 4 & 10 & $1.7(0.10)$ \\
\hline \multirow[t]{4}{*}{ LWM-2OH } & 2.5 & 1 & 2 & 1 & $6.5(0.25)$ \\
\hline & 5 & 2 & 2 & 1 & $5.5(0.30)$ \\
\hline & 10 & 2 & 3 & 1 & $4.4(0.30)$ \\
\hline & 20 & 2 & 2 & 0 & - \\
\hline \multirow[t]{3}{*}{ HWMH } & 2.5 & 36 & 19 & 10 & $0.5(0.03)$ \\
\hline & 5 & 28 & 15 & 9 & $0.5(0.09)$ \\
\hline & 10 & 6 & 4 & 6 & $2.0(0.05)$ \\
\hline \multirow[t]{2}{*}{ HMW-6OH } & 2.5 & 11 & 7 & 1 & $1.7(0.09)$ \\
\hline & 5 & 10 & 7 & 1 & $1.6(0.1)$ \\
\hline \multirow[t]{2}{*}{ HMW-2OH } & 2.5 & - & - & - & $>6 \mathrm{~h}$ \\
\hline & 2.5 & - & - & - & \\
\hline
\end{tabular}


$2 k_{+} m(t) P(t)$ represents fibril elongation by the addition of monomers to the two growing ends of the fibres. We considered minor modifications to these equations that might account for the critical involvement of the heparin cofactor, $h$, in the $\Delta$ tau187 nucleation and elongation processes, and which may also explain the influence of heparin on both aggregation rate and filament mass (Scheme 2). The trends observed can be rationalised if it is assumed that $\Delta$ tau187 monomers and heparin molecules rapidly establish an equilibrium

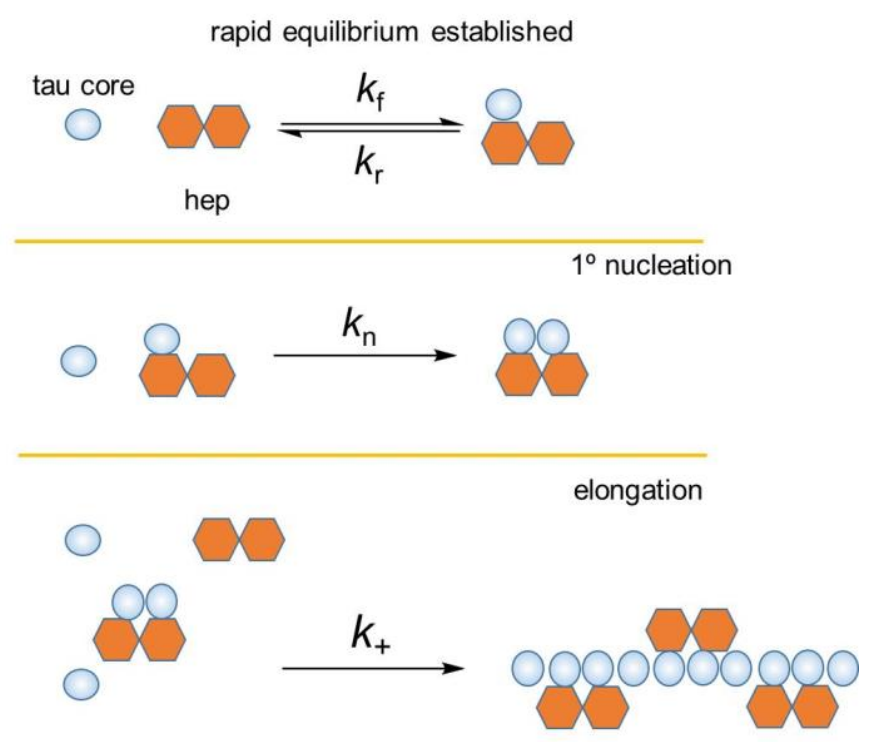

Scheme 2. Basic pathway for heparin-induced tau assembly into filaments.

state 1:1 complex $(\mathrm{mh})$. Neglecting depletion of monomer and heparin concentrations by other interaction pathways at this initial condition, the equilibrium concentration of the complex $[\mathrm{mh}]$ is, according to the standard relationship, given by

$$
[m h]=\frac{1}{2}\left\{K_{d}+[m]_{T}+[h]_{T}-\sqrt{\left(K_{d}+[m]_{T}+[h]_{T}\right)^{2}-4[m]_{T}[h]_{T}}\right\}
$$

where $[m] \mathrm{T}$ and $[h] \mathrm{T}$ are the total concentrations of free protein monomer and free heparin and $K_{d}$ is the dissociation constant. The equilibrium is perturbed as $m h$ recruits further monomers to form a nucleating species. Aggregation of the 4-repeat domain tau construct in the presence of heparin has been shown to be nucleation dependent, with a single heparin molecule binding tau forming an aggregationpromoting dimer, which then serves as a building block for further fibril growth. ${ }^{31} \mathrm{We}$ therefore assume that the smallest primary nucleating species $N$ consists of two tau molecules bound to one heparin molecule, $m_{2} h$. The rate of formation of primary 
nuclei $N$ thus depends on collisions between free monomeric protein and the $m h$ complex such that

$\frac{d N(t)}{d t}=k_{n} m h(t) m(t)^{n_{c}}$

where $n_{c}=1$. Equations [3] and [4] thus take into account the role of heparin in the initiation of tau aggregation. Equation [1] becomes

$\frac{d P(t)}{d t}=k_{2} m(t)^{n_{2}} M(t)+k_{n} m h(t) m(t)$

These equations likely oversimplify the tau-heparin interaction, but serve as a working hypothesis until the details of the model are further refined with experimental input. The simulated curves share similarities with the experimental data in Figure 2, the simulations do not replicate exactly the behaviour of the ThT curves and so quantitative analysis using global fitting of equations [4] and [5] to the curves was not attempted. However, qualitative inspection of numerically-simulated curves based on these adaptations suggest that $t_{0.5}$ for aggregation and filament mass are both sensitive to heparin concentration and to $K_{d}$ (Figure 3 ).

An equilibrium association constant exceeding $10^{6} \mathrm{M}^{-1}$ (i.e. $K_{d}<1 \mu \mathrm{M}$ ) for tau and heparin has been reported. ${ }^{30}$ According to Eq. [5], when $K_{d}=0.1 \mu \mathrm{M}$, to.5 for filament growth does not change markedly at heparin concentrations up to $10 \mu \mathrm{M}$, and the filament mass increases with heparin concentration up to $10 \mu \mathrm{M}$ (Figure 3 , top left panel). At a heparin concentration of $20 \mu \mathrm{M}$, a considerable increase in to.5 is predicted and the end-point filament mass decreases significantly. This apparent inhibitory effect at higher heparin concentrations can be rationalised as the highaffinity heparin-tau interaction removing free monomer from solution, leaving little remaining heparin to form nuclei and/or elongate the filaments. These trends in fibril mass and t0.5 are reminiscent of the ThT fluorescence curves for $\Delta$ tau187 with LMWH and LMW-2OH (Figure 2 and Table 1), although the latter curve is consistent with much slower aggregation than represented by the simulation. Hence, removal of the 2-O-sulfate group does not appear to reduce the affinity of heparin for $\Delta$ tau187 according to these curves. The slower rate of tau aggregation in the presence of $\mathrm{LMW}-2 \mathrm{OH}$, compared to the rates in the presence of the other heparin derivatives is reflected in the lower rate constants $k_{n}, k_{+}$and $k_{2}$ calculated by curve fitting (Figure 2 
and Table 1), which indicates that 2-O-sulfate group is critical for tau primary and secondary nucleation and filament elongation.
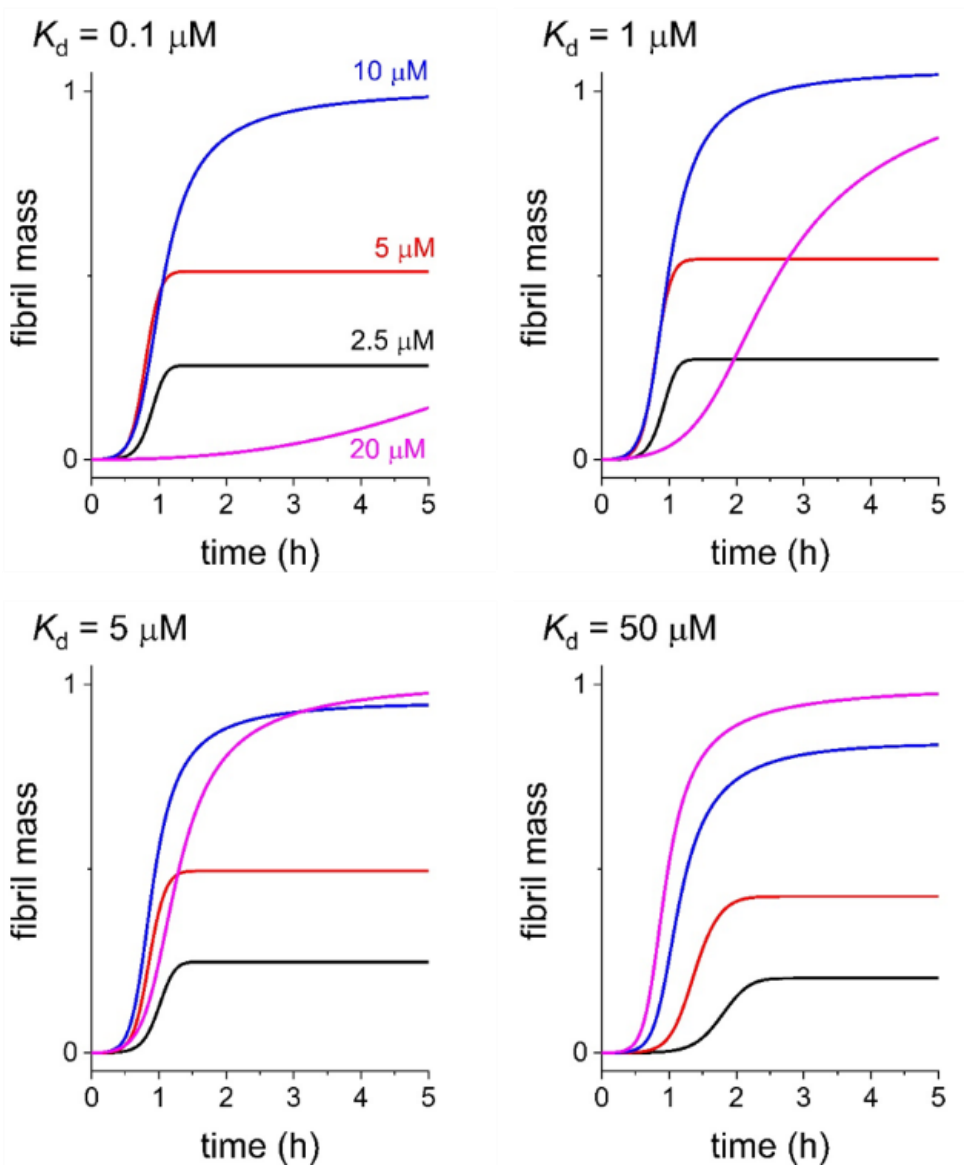

Figure 3. Calculated fibril mass at four heparin concentrations, based on the proposed model. The value of $K_{\mathrm{d}}$ for the initial tau-heparin complex is the only variable in the calculations. Using the terminology of Knowles and colleagues, values of the remaining constants are: $k_{n}=5 \times 10^{-4} \mathrm{M}^{-1} \mathrm{~s}^{-1}, k_{+}=8 \times 10^{6} \mathrm{M}^{-1} \mathrm{~s}^{-1}$. No fibril fragmentation of secondary nucleation (i.e., $k_{2}=0$ ) was assumed for simplification.

For progressively weaker tau-heparin interactions, the calculated fibril mass in the presence of $20 \mu \mathrm{M}$ heparin increases as $K_{d}$ increases (Figure 3). At $K_{d}=50 \mu \mathrm{M}$, a progressive decrease in $t_{0.5}$ and increase in fibril mass is predicted as the heparin concentration is increased. These trends are reminiscent of the ThT curves for tau in the presence of LMW-6OH (Figure 2, top right), and support the previous reports that 6-O-desulfation of heparin reduces its affinity for tau. ${ }^{19}$ Similarly, at $K_{d}=5 \mu \mathrm{M}$, the curves are reminiscent of the ThT profiles for tau in the presence of LMW-NAc, although there are also some important differences that indicate that our model is incomplete. 
Heparin 2-O-desulfation, but not 6-O-desulfation, affects the structure and morphology of $\Delta$ tau187 aggregates

We next used circular dichroism (CD) spectroscopy to follow the structural transformation accompanying $\Delta$ tau187 aggregation in the presence of $\mathrm{HMWH}, \mathrm{HMW}$ $6 \mathrm{OH}$ and $\mathrm{HMW}-2 \mathrm{OH}$. CD has indicated that tau aggregation accompanies a transition from an unfolded structure to a partially folded structure with approximately $\sim 36 \% \beta$ sheet. ${ }^{32}$ Here, we obtained spectra from freshly prepared $\Delta$ tau187-heparin solutions and again after incubation at $37^{\circ} \mathrm{C}$ for $8 \mathrm{~h}$. In the absence of heparin, the spectrum changes little over $8 \mathrm{~h}$ (Figure 4A), with a minimum at $200 \mathrm{~nm}$ consistent with the protein remaining unfolded, and a secondary structure content of $3.7( \pm 0.8) \%$ a-helices, $25( \pm$ $0.4) \% \beta$-sheet, $18( \pm 0.1) \%$ turns and $54( \pm 1.2) \%$ unordered. In the presence of unmodified heparin, $\Delta$ tau187 undergoes a structural transition over $8 \mathrm{~h}$ (Figure 4B, left). The spectrum at the end-point of aggregation with intact heparin is consistent with 0.7 ( \pm 1.3) \% a-helices, 46 ( \pm 11$) \% \beta$-sheet, 14 ( \pm 1.2$) \%$ turns and $39( \pm 9.3) \%$ unordered content, and an isodichroic point at around $208 \mathrm{~nm}$. A similar trend is seen in the CD spectra of $\Delta$ tau187 in the presence of 6-O-desulfated HMWH (Figure 4B, middle) with an increase in $\beta$-sheet content to $37( \pm 3.6) \%$ and no further change, indicating that aggregation has reached completion after $8 \mathrm{~h}$ with the structural content being similar to aggregates formed with unmodified heparin. By contrast, the spectrum of $\Delta$ tau187 in the presence of 2-O-desulfated HMWH (Figure 4B, right) changes little over $8 \mathrm{~h}$ and is consistent with $5.3( \pm 0.1) \%$ a-helices, $27( \pm 2.5) \%$-sheet, $17( \pm 0.5) \%$ turns and 51 ( \pm 1.8) \% unordered residues, closely resembling the structure content of monomeric $\Delta$ tau187 after 8 hours. Furthermore, there is no clear isodichroic point as seen in the spectra of tau with HMWH or LMW-6OH. Hence, the CD spectra concur with ThT measurements that the rate of $\Delta$ tau187 aggregation is slower in the presence of 2-Odesulfated heparin than with the other heparin derivatives. 


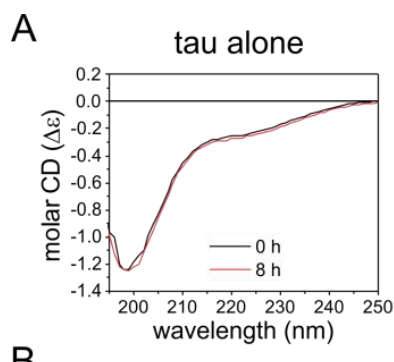

B

tau + HMWH
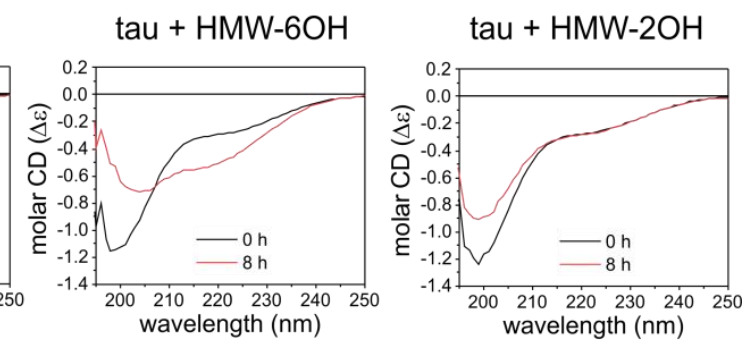

C
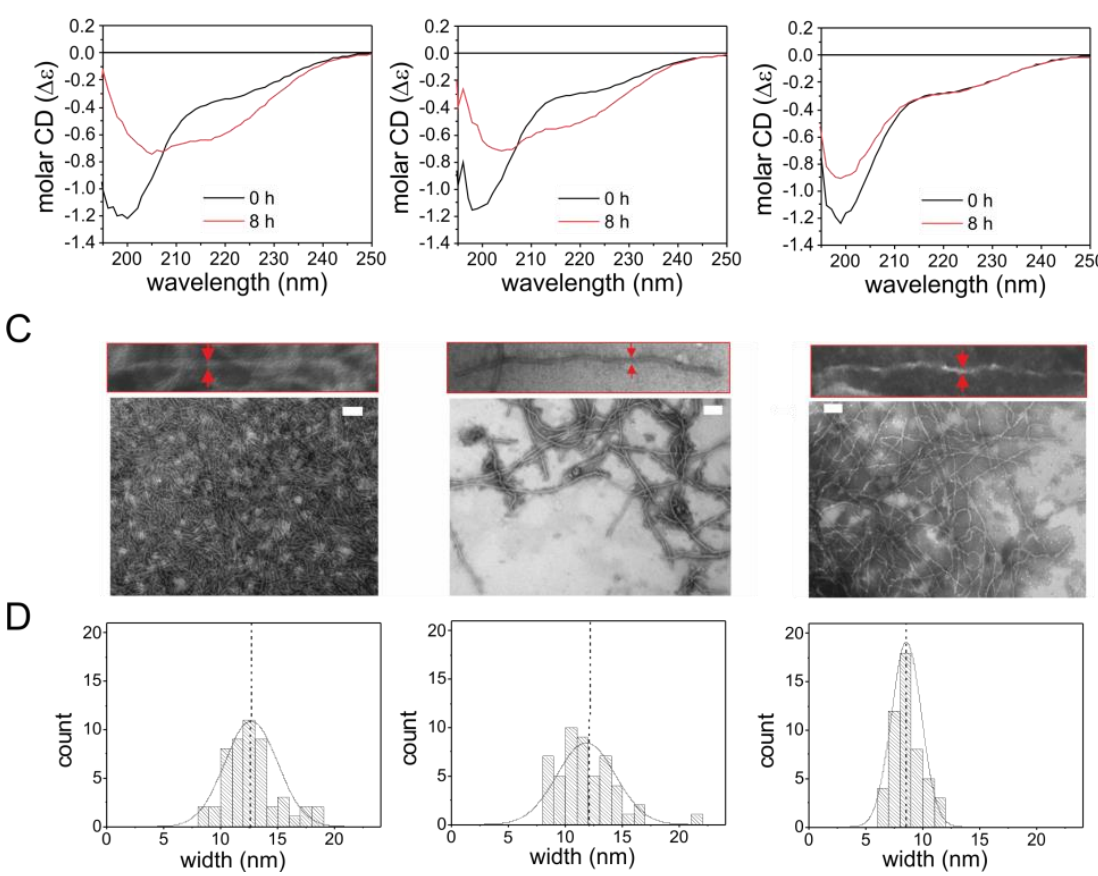

Figure 4. Secondary structure and morphology of $\Delta$ tau187 aggregates. (A) CD spectra of $\Delta$ tau187 $(20 \mu \mathrm{M})$ at $0 \mathrm{~h}$ and $8 \mathrm{~h}$. (B) CD spectra of $\Delta$ tau187 in the presence of $5 \mu \mathrm{M} \mathrm{HMWH,} \mathrm{6-O-desulfated} \mathrm{HMWH}$ or 2-O-desulfated $\mathrm{HMWH}$ at $0 \mathrm{~h}$ and $8 \mathrm{~h}$. The samples were incubated at $37^{\circ} \mathrm{C}$ without agitation. All CD spectra are shown as the average of triplicate repeats. (C) TEM images of aggregates formed from $\Delta$ tau187 $(20 \mu \mathrm{M})$ after incubating with $5 \mu \mathrm{M}$ of $\mathrm{HMWH}$ or the desulfated derivatives without agitation for $24 \mathrm{~h}$. Scale bar $=200 \mathrm{~nm}$. (D) Distribution of filament widths measured from the TEM images.

The morphology of aggregates formed after incubation with heparin or each of the desulfated analogues was visualised by negative-stain TEM. Four tau morphologies formed in the presence of heparin, termed snake, twister, hose and jagged, have been observed by cryo-EM. ${ }^{12}$ Here, in the presence of unmodified HMWH, $\Delta$ tau187 forms a dense network of fibrillar aggregates and upon closer inspection the filaments display a sinusoidal curvature pattern with a mean width of $11.8( \pm 5.2) \mathrm{nm}$ (Figure 4, C and D, left). The intertwined filaments that are characteristic of PHFs, or their crossover points, cannot be discerned here, but the width of the filaments is similar to that typically seen for paired filaments (10-25 nm). 5, 32-34 Aggregates formed in the presence of 6-O-desulfated 
heparin display a similar morphology and a mean width of $12.7( \pm 4.6)$ (Figure 4, C and $\mathrm{D}$, middle), although their sparse deposition is consistent with a lower yield of filaments. Filaments formed in the presence of HMW-2OH (Figure 4C, right) are also sparsely distributed and display a sinusoidal curvature pattern. These filaments are noticeably more slender than the filaments formed with unmodified heparin, with mean width of 8.5 $( \pm 2.6) \mathrm{nm}$ (Figure $4 \mathrm{D}$, right). The width of these filaments is more typical of the single filaments that are the minor population of tau aggregates associated with AD. 33,34 To summarise, heparin 2-O-desulfation has a much more profound effect on tau aggregation rate, filament mass, $\beta$-sheet content and filament morphology than either 6 O-desulfation or $\mathrm{N}$-acetylation.

\section{Tau filaments formed with 2-O-desulfated heparin have a reduced $\beta$-sheet core}

Finally, we used magic-angle spinning (MAS) solid-state NMR (SSNMR) to compare the structure and dynamics of uniformly ${ }^{13} \mathrm{C}$-labeled $\Delta$ tau187 fibrils formed in the presence of $\mathrm{HWMH}$ and HMW-2OH. Previous SSNMR analyses of heparin-induced aggregates of truncated tau constructs indicated that amino acids 306-324 form the rigid $\beta$-sheet core and adopt two distinct conformations ${ }^{7,8}$ consistent with the polymorphism of tau filaments visualised by cryo-EM. ${ }^{12}$ Unlike electron microscopy, the flexible $\mathrm{N}$ - and C-terminal sequences constituting the "fuzzy" regions may be observed by MAS SSNMR using INEPT-based methods that correlate nuclear spins through $J$-couplings, while the ordered core is observed selectively using Hartmann-Hahn cross-polarization combined with nuclear dipolar recoupling methods. ${ }^{32,35}$

Here, two-dimensional ${ }^{13} \mathrm{C}-{ }^{13} \mathrm{C}$ dipolar correlation NMR spectra were obtained from fibrils formed by $\Delta$ tau187 $(20 \mu \mathrm{M})$ in the presence of $5 \mu \mathrm{M} \mathrm{HMWH}$ or HMW-2OH. By using a short dipolar mixing time $(10 \mathrm{~ms})$, the spectra report on the motionallyrestrained residues forming the core of the fibrils. The more flexible outer residues give rise to weaker cross-peaks or are not observed. The peaks are quite broad, which probably reflects the known structural heterogeneity of tau filaments formed in the presence of heparin. ${ }^{13}$ No attempt was made to sequentially assign the spectra owing to severe crowding and overlap, as the tau construct we studied is much larger than the constructs 

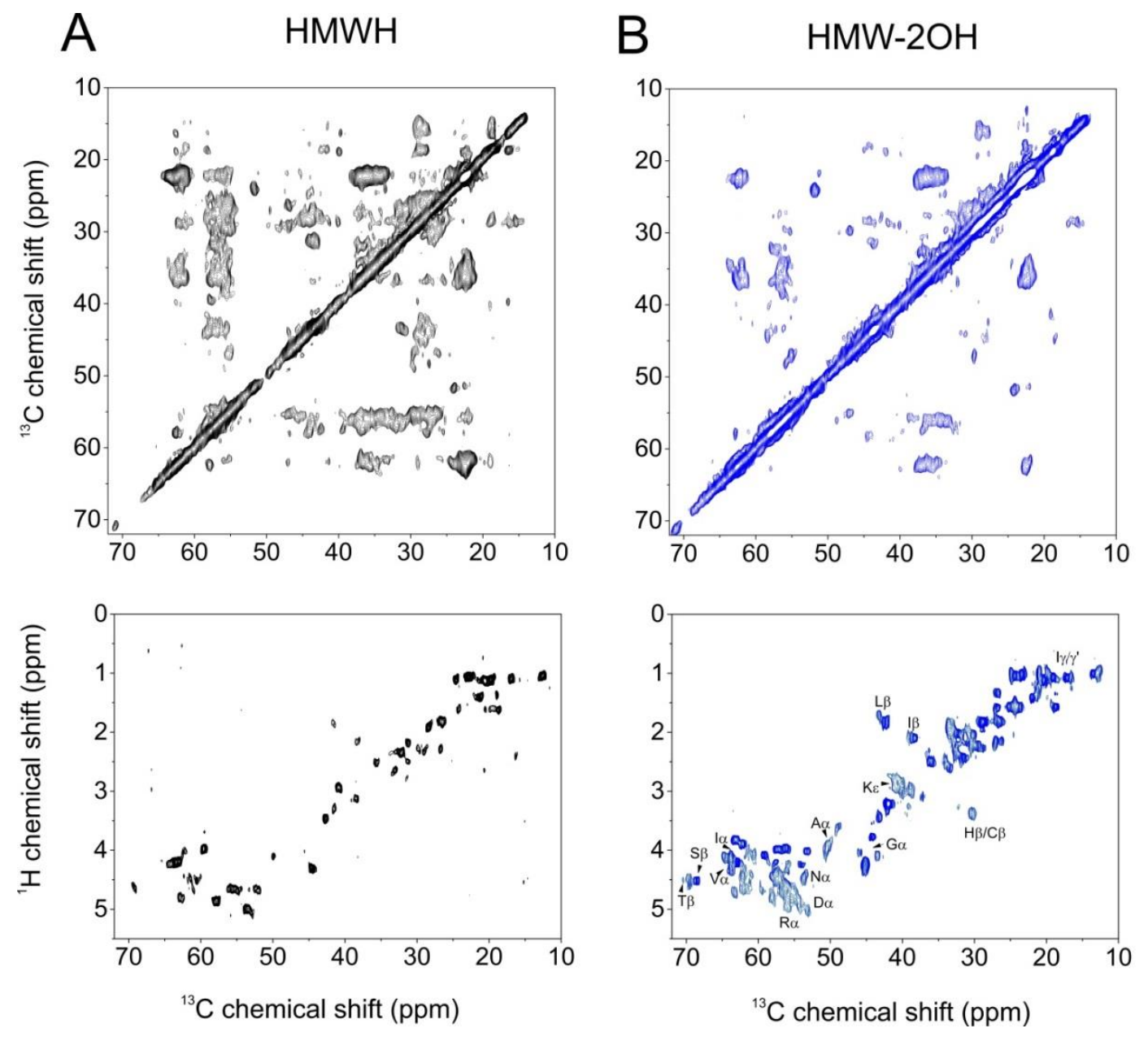

Figure 5. Solid-state magic-angle spinning ${ }^{13} \mathrm{C}$ NMR spectra of uniformly ${ }^{13} \mathrm{C}$-labelled $\Delta$ tau187 aggregates formed in the presence of $\mathrm{HMWH}$ or $6-\mathrm{O}$ - or 2-O- desulfated HMWH derivatives. (A) Top: region of a ${ }^{13} \mathrm{C}-{ }^{13} \mathrm{C}$ dipolar correlation NMR spectrum of $\left[\mathrm{U}-{ }^{13} \mathrm{C}\right] \Delta$ tau187 aggregates formed in the presence of $\mathrm{HMWH}$. Dipolar interactions were reintroduced by 5 ms DARR mixing period. Bottom: $A{ }^{1} \mathrm{H}-{ }^{13} \mathrm{C}$ INEPT spectrum of the sample sample (B) The ${ }^{13} \mathrm{C}-{ }^{13} \mathrm{C}$ dipolar correlation NMR spectrum and INEPT spectrum of tau filaments formed in the presence of $\mathrm{HMW}-2 \mathrm{OH}$. The MAS frequency was $13 \mathrm{kHz}$ in all experiments.

previously studied by SSNMR. Sequential assignment and structural determination could in principle be achieved with a combination of $2 \mathrm{D}$ and $3 \mathrm{D}$ NMR experiments and selective isotope labelling, but this was beyond the scope of the present work. The experimental spectrum of tau filaments formed with $\mathrm{HMWH}$ is overlaid with a simulated spectrum (Supporting Information, Figure S1) generated from ${ }^{13} \mathrm{C}$ chemical shifts predicted from the cryo-EM structure of heparin-induced filaments (Table S1), ${ }^{12}$ which are predominantly $\beta$-sheet/hairpin folds. Good agreement between the simulated and experimental spectra suggests that the observed signals originate from the $\beta$-sheet core of the fibrils. A striking reduction in cross-peak intensities, particularly for cross-peaks 
representing longer-range couplings, is seen in the spectrum of the fibrils induced by $\mathrm{HMW}-2 \mathrm{OH}$, as compared to the spectrum of fibrils induced by HMWH (Figure 5, A and $\mathrm{B}$, top panels). The reduction in intensity is consistent with a higher degree of overall flexibility within the fibrils induced by $\mathrm{HMW}-2 \mathrm{OH}$. In contrast to the ${ }^{13} \mathrm{C}-{ }^{13} \mathrm{C}$ spectra, ${ }^{1} \mathrm{H}$ ${ }^{13} \mathrm{C}$ INEPT spectra of the exhibit many more peaks than are seen than in the spectrum of $\mathrm{HMWH}$-induced filaments (Figure 5, A and B, bottom panels), confirming that the HMW$2 \mathrm{OH}$-induced fibrils contain a greater number of flexible residues than do fibrils induced by $\mathrm{HMWH}$. In the INEPT spectrum of HMW-2OH-induced filaments, much stronger signals are observed from methyl bearing side chains, but also in the $\mathrm{C} \alpha$ region, indicating that more residues are flexible in the protein backbone. Taken together, these observations are consistent with the $\mathrm{HMW}-2 \mathrm{OH}$-induced filaments having a smaller $\beta$ sheet core, flanked by a greater number of mobile residues, compared to the filaments formed in the presence of unmodified heparin.

\section{Discussion}

Heparin is a convenient experimental aid to induce the rapid formation of tau filaments/fibrils in vitro for mechanistic and structural investigations. It is a close analog of the GAG heparan sulfate, and heparan sulfate proteoglycans (HSPGs) such as agrin are commonly associated with $A \beta$ plaques and NFTs in AD. The sulfation patterns of GAGs are known to change with ageing, ${ }^{36,37}$ the principal riskfactor for $A D$, and so there is biological incentive to investigate how GAG sulfation affects tau aggregation, using heparin as a representative GAG.

Experimental evidence gained over the last decade suggests that the progression of neurodegeneration in $A D$ is driven by the transcellular propagation of tau aggregates, which seed protein aggregation in the recipient cells in a prion-like manner. ${ }^{38,} 39$ This process may not necessarily be limited to tau aggregates, as monomeric tau can also be internalised and seed the aggregation of endogenous tau. 40 The mechanism of tau release from neurons into the extracellular space is not currently known, but the cellular uptake of tau fibrils can occur via binding to HSPGs. ${ }^{15}$ It is conceivable that HSPGs also promote the fibrillisation of tau monomers and oligomers in the extracellular space, just as heparin (and HS) do in vitro, in addition to facilitating the cellular uptake of fibrils. 
Here we report that selectively altering the sulfation pattern of heparin affects the kinetics of heparin-induced $\Delta$ tau187 aggregation and the mass of filaments formed, and can also modify the molecular structure of the filaments. The rate of aggregation is slower in the presence of all the desulfated heparins than in the presence of native heparin, which may be attributed in part to the lower negative charge of the derivatives. All the heparins desulfated at a single position have approximately the same overall sulfation levels, however, and the differences in the behaviour of tau in the presence of 6-O-desulfated, 2-O-desulfated and $\mathrm{N}$-acetylated heparins suggest that the derivatives have distinctive chemical properties that modulate their interactions with tau.

We propose a basic mechanism for heparin-induced tau aggregation, which goes some way toward explaining the aggregation kinetics in the presence of the different heparin derivatives, but significant differences between the experimental ThT curves and calculated curves indicate that the model is incomplete. A more robust analysis may need to take into account the formation of oligomers, polymerization via multiple pathways and complex secondary nucleation processes involving protein fibrillar and heparin surfaces. As it stands, the basic model is consistent with the 6-O-desulfated and, to a lesser extent, $\mathrm{N}$-desulfated heparin having lower affinity for tau than intact heparin. This interpretation agrees with previous work: using a heparin-immobilized chip, surface plasmon resonance was used to show that $\mathrm{N}$-desulfation and 2-O-desulfation had no effect on heparin binding to a tau construct, whereas 6 -O-desulfation severely reduced binding. ${ }^{19} \mathrm{In}$ addition, cellular studies have shown that heparin interacts with heparan sulfate binding sites in tau and prevents binding to cell-surface HSPGs, ${ }^{15}$ but removal of the 6-O-sulfates from heparin abolishes the inhibitory effect whereas 2-O-desulfated heparin remains strongly inhibitory. ${ }^{17}$ Further studies concur that 6 -O-sulfation is critical for tau-heparan sulfate interactions and that this modification regulates uptake in human cell lines and mouse brain slice culture. ${ }^{18}$ Interestingly, the 6-OS and NS glucosamine sulfates, but not the 2-OS iduronate sulfate, of heparin is required for binding to $A \beta$ fibrils, ${ }^{41}$ suggesting a common function for this moiety in amyloid binding.

The most interesting finding here is that 2-O-desulfation of heparin results in a markedly greater reduction of the $\Delta$ tau187 aggregation rate than does desulfation at 
the other two sites, and templates tau assembly into a different filament structure and morphology. These differences may be rationalised by considering the effects of removing sulfate groups on the conformation of heparin. In addition to losing a charge in the form of a sulfate group, the conformation of heparin derivatives is sensitive to the substitution pattern in several ways. The iduronate residue is known to be in an equilibrium of ${ }^{1} \mathrm{C}^{4}$ and ${ }^{2} \mathrm{~S}_{0}$ chair and skew boat forms in heparin 67:33 $\left.{ }^{1} \mathrm{C}^{4: 2} \mathrm{~S}_{0}\right)^{42}$ but, following de-O-sulfation at position-2 of the iduronate residue, this equilibrium is disturbed, moving in favour of the ${ }^{1} \mathrm{C}_{4}$ chair form $(85: 15)^{43}$. In addition, removal of 2-O-sulfate groups alters the ${ }^{1} \mathrm{JCH}$ NMR coupling constants $(\mathrm{C}-1$ to $\mathrm{H}-1$ and $\mathrm{C}-4$ to $\mathrm{H}-4$ ) around the glycosidic linkages, consistent with additional changes in conformation, ${ }^{44}, 45$ for example, the ${ }^{1} \mathrm{~J} \mathrm{CH}$ values change at A-1 (C-1 to $\mathrm{H}-1$ of glucosamine) from 172 to $176 \mathrm{~Hz}$ and at $\mathrm{I}-1$ (C-1 to $\mathrm{H}-1$ of the iduronate residue) from 147 to $156 \mathrm{~Hz} .{ }^{46}$ The removal of 6-O-sulfate groups from glucosamine, on the other hand, while altering chemical shift values principally adjacent to the site of the sulfation as expected, ${ }^{24}$ has less effect elsewhere in the heparin chain, consistent with relatively little perturbation of the overall conformation. ${ }^{47}$ We propose that the unique conformational perturbations resulting from 2-O-desulfation must therefore modify the tau-heparin interaction in such a way that the conformation of tau is less amenable to self-assembly. One explanation, which is supported by the NMR data, is that the core $\beta$-sheet region of tau is reduced and the flanking fuzzy coat region extends further into the core. Why a different filament structure and morphology occurs in the presence of 2-O-desulfated heparin can only be speculated upon at this stage. Alterations in the global and local conformation of heparin after 2-Odesulfation may reduce or otherwise change the charged surface that interacts with tau monomers. A distinct primary nucleating species may be formed that directs propagation along a structurally and kinetically altered pathway involving interactions between fewer core residues.

There is ample evidence that heparin sulfates accelerate the aggregation of monomeric tau in vitro. ${ }^{48}$ Further work will be necessary to understand fully the nature of the tau species that interact with HSPGs during transcellular propagation, and to ascertain whether GAGs play a role in filament formation. It should be noted, that protein aggregates in the Alzheimer's brain principally contains phosphorylated tau (P-tau), rather than normal tau, and as such the higher negative charge density 
would likely repel polyanionic GAGs. The interplay between tau, P-tau and GAGs must therefore be considered in future investigations. The key message of this work is that to understand the role of GAG-protein interactions in the formation of amyloid fibrils, it is necessary to look beyond electrostatics and to consider the chemical and conformational effects conferred on GAGs by their sulfation patterns.

\section{Acknowledgments}

Funding was provided by BBSRC (UK) Grant BB/K015958/1.

\section{Supporting Information}

One figure and one table.

\section{References}

[1] Gibbons, G. S., Lee, V. M. Y., and Trojanowski, J. Q. (2019) Mechanisms of Cellto-Cell Transmission of Pathological Tau a Review, Jama Neurology 76, 101108.

[2] Guo, T., Noble, W., and Hanger, D. P. (2017) Roles of Tau Protein in Health and Disease, Acta Neuropathol. 133, 665-704.

[3] Goedert, M., Spillantini, M. G., Jakes, R., Rutherford, D., and Crowther, R. A. (1989) Multiple Isoforms of Human Microtubule-Associated Protein-Tau: Sequences and Localization in Neurofibrillary Tangles of Alzheimer's Disease, Neuron 3, 519-526.

[4] Iqbal, K., Alonso, A. D. C., Chen, S., Chohan, M. O., El-Akkad, E., Gong, C. X., Khatoon, S., Li, B., Liu, F., Rahman, A., Tanimukai, H., and Grundke-lqbal, I. (2005) Tau Pathology in Alzheimer Disease and Other Tauopathies, Biochimica Et Biophysica Acta-Molecular Basis of Disease 1739, 198-210.

[5] Fitzpatrick, A. W. P., Falcon, B., He, S., Murzin, A. G., Murshudov, G., Garringer, H. J., Crowther, R. A., Ghetti, B., Goedert, M., and Scheres, S. H. W. (2017) Cryo-Em Structures of Tau Filaments from Alzheimer's Disease, Nature 547, 185-190.

[6] Wegmann, S., Medalsy, I. D., Mandelkow, E., and Muller, D. J. (2013) The Fuzzy Coat of Pathological Human Tau Fibrils Is a Two-Layered Polyelectrolyte Brush, Proc. Natl. Acad. Sci. U. S. A. 110, E313-E321. 
[7] Daebel, V., Chinnathambi, S., Biernat, J., Schwalbe, M., Habenstein, B., Loquet, A., Akoury, E., Tepper, K., Muller, H., Baldus, M., Griesinger, C., Zweckstetter, M., Mandelkow, E., Vijayan, V., and Lange, A. (2012) BetaSheet Core of Tau Paired Helical Filaments Revealed by Solid-State Nmr, J. Am. Chem. Soc. 134, 13982-13989.

[8] Andronesi, O. C., von Bergen, M., Biernat, J., Seidel, K., Griesinger, C., Mandelkow, E., and Baldus, M. (2008) Characterization of Alzheimer's-Like Paired Helical Filaments from the Core Domain of Tau Protein Using SolidState Nmr Spectroscopy, J. Am. Chem. Soc. 130, 5922-5928.

[9] Falcon, B., Zhang, W. J., Murzin, A. G., Murshudov, G., Garringer, H. J., Vidal, R., Crowther, R. A., Ghetti, B., Scheres, S. H. W., and Goedert, M. (2018) Structures of Filaments from Pick's Disease Reveal a Novel Tau Protein Fold, Nature 561, 137-140.

[10] Fichou, Y., Lin, Y. X., Rauch, J. N., Vigers, M., Zeng, Z. K., Srivastava, M., Keller, T. J., Freed, J. H., Kosik, K. S., and Han, S. I. (2018) Cofactors Are Essential Constituents of Stable and Seeding-Active Tau Fibrils, Proc. Natl. Acad. Sci. U. S. A. 115, 13234-13239.

[11] Perez, M., Valpuesta, J. M., Medina, M., deGarcini, E. M., and Avila, J. (1996) Polymerization of Tau into Filaments in the Presence of Heparin: The Minimal Sequence Required for Tau-Tau Interaction, J. Neurochem. 67, 1183-1190. [12] Zhang, W. J., Falcon, B., Murzin, A. G., Fan, J., Crowther, R. A., Goedert, M., and Scheres, S. H. W. (2019) Heparin-Induced Tau Filaments Are Polymorphic and Differ from Those in Alzheimer's and Pick's Diseases, Elife 8: e43584.

[13] Fichou, Y., Vigers, M., Goring, A. K., Eschmann, N. A., and Han, S. I. (2018) Heparin-Induced Tau Filaments Are Structurally Heterogeneous and Differ from Alzheimer's Disease Filaments, Chem. Commun. 54, 4573-4576.

[14] Snow, A. D., Mar, H., Nochlin, D., Sekiguchi, R. T., Kimata, K., Koike, Y., and Wight, T. N. (1990) Early Accumulation of Heparan-Sulfate in Neurons and in the Beta-Amyloid Protein-Containing Lesions of Alzheimer's Disease and Down's Syndrome, Am. J. Pathol. 137, 1253-1270.

[15] Holmes, B. B., DeVos, S. L., Kfoury, N., Li, M., Jacks, R., Yanamandra, K., Ouidja, M. O., Brodsky, F. M., Marasa, J., Bagchi, D. P., Kotzbauer, P. T., Miller, T. M., Papy-Garcia, D., and Diamond, M. I. (2013) Heparan Sulfate 
Proteoglycans Mediate Internalization and Propagation of Specific

Proteopathic Seeds, Proc. Natl. Acad. Sci. USA 110, E3138-E3147.

[16] Capila, I., and Linhardt, R. J. (2002) Heparin - Protein Interactions, Angew.

Chem. Int. Ed. 41, 391-412.

[17] Stopschinski, B. E., Holmes, B. B., Miller, G. M., Manon, V. A., Vaquer-Alicea,

J., Prueitt, W. L., Hsieh-Wilson, L. C., and Diamond, M. I. (2018) Specific Glycosaminoglycan Chain Length and Sulfation Patterns Are Required for Cell Uptake of Tau Versus Alpha-Synuclein and Beta-Amyloid Aggregates, J. Biol. Chem. 293, 10826-10840.

[18] Rauch, J. N., Chen, J. J., Sorum, A. W., Miller, G. M., Sharf, T., See, S. K., Hsieh-Wilson, L. C., Kampmann, M., and Kosik, K. S. (2018) Tau Internalization Is Regulated by 6-O Sulfation on Heparan Sulfate Proteoglycans (HSPGs), Sci. Rep. 8:6382.

[19] Zhao, J., Huvent, I., Lippens, G., Eliezer, D., Zhang, A. Q., Li, Q. H., Tessier, P., Linhardt, R. J., Zhang, F. M., and Wang, C. Y. (2017) Glycan Determinants of Heparin-Tau Interaction, Biophys. J. 112, 921-932.

[20] Hasegawa, M., Smith, M. J., and Goedert, M. ( 1998) Tau Proteins with Ftdp-17 Mutations Have a Reduced Ability to Promote Microtubule Assembly, FEBS Lett. 437, 207-210.

[21] Inoue, Y., and Nagasawa, K. (1976) Selective N-Desulfation of Heparin with Dimethyl Sulfoxide Containing Water or Methanol, Carbohydr. Res. 46, 87-95.

[22] Nagasawa, K., Inoue, Y., and Kamata, T. (1977) Solvolytic Desulfation of Glycosaminoglycuronan Sulfates with Dimethyl-Sulfoxide Containing Water or Methanol Carbohydr. Res. 58, 47-58.

[23] Jaseja, M., Rej, R., Sauriol, F., and Perlin, A. (1989) Novel Regioselective and

Stereoselective Modifications of Heparin in Alkaline Solution - Nuclear

Magnetic Resonance Spectroscopic Evidence Can. J. Chem. 67, 1449-1456.

[24] Yates, E. A., Santini, F., Guerrini, M., Naggi, A., Torri, G., and Casu, B. (1996)

H-1 and C-13 Nmr Spectral Assignments of the Major Sequences of Twelve Systematically Modified Heparin Derivatives, Carbohydr. Res. 294, 15-27. [25] Micsonai, A., Wien, F., Bulyáki, É., Kun, J., Moussong, É., Lee, Y.-H., Goto, Y., Réfrégiers, M., and Kardos, J. (2018) Bestsel: A Web Server for Accurate Protein Secondary Structure Prediction and Fold Recognisiton from the Circular Dichorism Spectra, Nucleic Acids Res. 46 (W1), W315-W322. 
[26] Takegoshi, K., Nakamura, S., and Terao, T. (2001) C-13-H-1 Dipolar-Assisted Rotational Resonance in Magic-Angle Spinning Nmr, Chem. Phys. Lett. 344, 631-637.

[27] Cohen, S. I. A., Linse, S., Luheshi, L. M., Hellstrand, E., White, D. A., Rajah, L., Otzen, D. E., Vendruscolo, M., Dobson, C. M., and Knowles, T. P. J. (2013) Proliferation of Amyloid-Beta 42 Aggregates Occurs through a Secondary Nucleation Mechanism, Proc. Natl. Acad. Sci. U. S. A. 110, 9758-9763.

[28] Knowles, T. P. J., Waudby, C. A., Devlin, G. L., Cohen, S. I. A., Aguzzi, A., Vendruscolo, M., Terentjev, E. M., Welland, M. E., and Dobson, C. M. (2009) An Analytical Solution to the Kinetics of Breakable Filament Assembly, Science 326, 1533-1537.

[29] Friedhoff, P., Schneider, A., Mandelkow, E. M., and Mandelkow, E. (1998) Rapid Assembly of Alzheimer-Like Paired Helical Filaments from MicrotubuleAssociated Protein Tau Monitored by Fluorescence in Solution, Biochemistry 37, 10223-10230.

[30] Zhu, H. L., Fernandez, C., Fan, J. B., Shewmaker, F., Chen, J., Minton, A. P., and Liang, Y. (2010) Quantitative Characterization of Heparin Binding to Tau Protein: Implication for Inducer-Mediated Tau Filament Formation., J. Biol. Chem. 285, 3592-3599.

[31] Ramachandran, G., and Udgaonkar, J. B. (2011) Understanding the Kinetic Roles of the Inducer Heparin and of Rod-Like Protofibrils During Amyloid Fibril Formation by Tau Protein, J. Biol. Chem. 286, 38948-38959.

[32] Barghorn, S., Davies, P., and Mandelkow, E. (2004) Tau Paired Helical Filaments from Alzheimer's Disease Brain and Assembled in Vitro Are Based on Beta-Structure in the Core Domain, Biochemistry 43, 1694-1703.

[33] Ksiezak-Reding, H., and Wall, J. S. (2005) Characterization of Paired Helical Filaments by Scanning Transmission Electron Microscopy, Microsc. Res. Tech. 67, 126-140.

[34] Nizynski, B., Dzwolak, W., and Nieznanski, K. (2017) Amyloidogenesis of Tau Protein, Protein Sci. 26, 2126-2150.

[35] Andronesi, O. C., Becker, S., Seidel, K., Heise, H., Young, H. S., and Baldus, M. (2005) Determination of Membrane Protein Structure and Dynamics by MagicAngle-Spinning Solid-State Nmr Spectroscopy, J. Am. Chem. Soc. 127, 12965-12974. 
[36] Foscarin, S., Raha-Chowdhury, R., Fawcett, J. W., and Kwok, J. C. F. (2017) Brain Ageing Changes Proteoglycan Sulfation, Rendering Perineuronal Nets More Inhibitory, Aging-US 9, 1607-1622.

[37] Feyzi, E., Saldeen, T., Larsson, E., Lindahl, U., and Salmivirta, M. (1998) AgeDependent Modulation of Heparan Sulfate Structure and Function, J. Biol. Chem. 273, 13395-13398.

[38] Kfoury, N., Holmes, B. B., Jiang, H., Holtzman, D. M., and Diamond, M. I. (2012)

Trans-Cellular Propagation of Tau Aggregation by Fibrillar Species, J. Biol. Chem. 287, 19440-19451.

[39] Iba, M., Guo, J. L., McBride, J. D., Zhang, B., Trojanowski, J. Q., and Lee, V. M.

Y. (2013) Synthetic Tau Fibrils Mediate Transmission of Neurofibrillary

Tangles in a Transgenic Mouse Model of Alzheimer's-Like Tauopathy, J.

Neurosci. 33, 1024-1037.

[40] Michel, C. H., Kumar, S., Pinotsi, D., Tunnacliffe, A., St George-Hyslop, P., Mandelkow, E., Mandelkow, E.-M., Kaminski, C. F., and Schierle, G. S. K. (2014) Extracellular Monomeric Tau Protein Is Sufficient to Initiate the Spread of Tau Protein Pathology, J. Biol. Chem. 289, 956-967.

[41] Stewart, K. L., Hughes, E., Yates, E. A., Middleton, D. A., and Radford, S. E. (2017) Molecular Origins of the Compatibility between Glycosaminoglycans and a Beta 40 Amyloid Fibrils, J. Mol. Biol. 429, 2449-2462.

[42] Hricovini, M., and Hricovini, M. (2018) Solution Conformation of Heparin

Tetrasaccharide. Dft Analysis of Structure and Spin-Spin Coupling Constants, Molecules 23:3042.

[43] Rudd, T. R., Guimond, S. E., Skidmore, M. A., Duchesne, L., Guerrini, M., Torri, G., Cosentino, C., Brown, A., Clarke, D. T., Turnbull, J. E., Fernig, D. G., and Yates, E. A. (2007) Influence of Substitution Pattern and Cation Binding on Conformation and Activity in Heparin Derivatives, Glycobiology 17, 983-993. [44] Hricovini, M., and Tvaroska, I. (1990) Conformation Dependence of Th EoneBond Carbon Proton Coupling-Constants in Oligosaccharides, Magn. Reson. Chem. 28, 862-866.

[45] Tvaroska, I., and Taravel, F. R. (1992) One-Bond Carbon Proton Coupling Constants - Angular Dependence in Beta-Linked Oligosaccharides, J. Biomol. NMR 2, 421-430. 
[46] Yates, E. A., Santini, F., De Cristofano, B., Payre, N., Cosentino, C., Guerrini, M., Naggi, A., Torri, G., and Hricovini, M. (2000) Effect of Substitution Pattern on $\mathrm{H}-1, \mathrm{C}-13 \mathrm{Nmr}$ Chemical Shifts and (1) J(Ch) Coupling Constants in Heparin Derivatives, Carbohydr. Res. 329, 239-247.

[47] Rudd, T. R., and Yates, E. A. (2010) Conformational Degeneracy Restricts the Effective Information Content of Heparan Sulfate, Mol. Biosyst. 6, 902-908. [48] Maiza, A., Chantepie, S., Vera, C., Fifre, A., Huynh, M. B., Stettler, O., Ouidja, M. O., and Papy-Garcia, D. (2018) The Role of Heparan Sulfates in Protein Aggregation and Their Potential Impact on Neurodegeneration, FEBS Lett. 592, 3806-3818. 
Accession identifier

Full length human MAPT: UniProtKB P10636 